\title{
Resolutions of the Coulomb operator: II. The Laguerre generator
}

\author{
Peter M.W. Gill *, Andrew T.B. Gilbert \\ Research School of Chemistry, Australian National University, Science Road, Canberra ACT 0200, Australia
}

\section{A R T I C L E I N F O}

\section{Article history:}

Received 11 August 2008

Accepted 16 October 2008

Available online 5 November 2008

\section{PACS:}

02.30.Em

02.70. $\mathrm{Hm}$

31.15.-p

41.20.CV

Keywords:

Coulomb operator

Cholesky decomposition

Kronecker product approximation

Resolution of the identity

Auxiliary basis sets

Density fitting

\begin{abstract}
A B S T R A C T
We discuss a resolution of the Coulomb operator, $r_{12}^{-1}=\left|\phi_{i}\right\rangle\left\langle\phi_{i}\right|$, into a one-particle basis. We show that the Laguerre polynomials generate a resolution with attractive computational properties and we apply it to the calculation of Coulomb and exchange energies in hydrogenic ions, the $\mathrm{H}_{2}$ molecule, and the Be atom. Rapid convergence is observed in all cases and a theoretical reason for this is discussed.
\end{abstract}

(c) 2008 Elsevier B.V. All rights reserved.

\section{Introduction}

The most troublesome terms in the non-relativistic Schrödinger Hamiltonians for atomic and molecular systems are the twoelectron Coulomb operators $r_{i j}^{-1}$. They are responsible for almost all of the difficulties that confront chemical physicists who seek to understand molecular electronic structure and the reason is simple: it is these terms that are responsible for the coupling of the electrons' motions and which thereby create many-body effects. Life would be much simpler - although life would almost certainly cease to exist - if these terms were removed.

Of course, we are not at liberty simply to drop such terms from our Hamiltonians, but one can ask whether they may be recast into a form that will facilitate both understanding and computation. This is the goal of the present paper and, in particular, we will examine the operator resolution

$r_{12}^{-1}=\left|\phi_{k}\right\rangle\left\langle\phi_{k}\right|$

(summation convention implied) and the associated function expansion

\footnotetext{
* Corresponding author.

E-mail address: peter.gill@anu.edu.au (P.M.W. Gill).
}

$r_{12}^{-1}=\sum_{k}^{\infty} \phi_{k}\left(\boldsymbol{r}_{1}\right) \phi_{k}\left(\boldsymbol{r}_{2}\right)$

which express the Coulomb operator as an infinite sum of binary products of one-electron operators.

A corollary of such a resolution is that we can write

$\left\langle a\left|r_{12}^{-1}\right| b\right\rangle=\left\langle a \mid \phi_{k}\right\rangle\left\langle\phi_{k} \mid b\right\rangle$

and thus reduce two-electron integrals to sums of products of overlap integrals. This expansion is exact but, if the sum over $k$ is truncated after a finite number of terms, it becomes reminiscent of the low-rank Cholesky [1-3] and Kronecker [4] approximations that are gaining popularity in quantum chemistry. An advantage of our method over other density fitting procedures such as resolution of the identity [5-8] and Poisson fitting $[9,10]$ is that we do not need to compute and manipulate the metric matrix that arises from the interactions between the auxiliary basis functions.

In the early 19 th century, a partial resolution was achieved by combining the Legendre expansion with the Addition Theorem for spherical harmonics to find

$$
\begin{aligned}
r_{12}^{-1} & =\left(r_{1}^{2}+r_{2}^{2}-2 r_{1} r_{2} \cos \gamma\right)^{-1 / 2}=\sum_{l=0}^{\infty} \frac{r_{<}^{l}}{r_{>}^{l+1}} P_{l}(\cos \gamma) \\
& =\frac{4 \pi}{2 l+1} \sum_{l=0}^{\infty} \sum_{m=-l}^{l} \frac{r_{<}^{l}}{r_{>}^{l+1}} Y_{l m}\left(\boldsymbol{r}_{1}\right) Y_{l m}\left(\boldsymbol{r}_{2}\right)
\end{aligned}
$$


where $\gamma$ is the angle between $\boldsymbol{r}_{1}$ and $\boldsymbol{r}_{2}$, and $Y_{l m}(\boldsymbol{r})$ is a spherical harmonic [11] of the angular part of $\boldsymbol{r}$. However, although this venerable expansion is very useful, it resolves $r_{12}^{-1}$ into functions of $\boldsymbol{r}_{<}$and $\boldsymbol{r}_{>}$(the lesser and greater of $\boldsymbol{r}_{1}$ and $\boldsymbol{r}_{2}$ ), rather than $\boldsymbol{r}_{1}$ and $\boldsymbol{r}_{2}$ themselves.

A decade ago, we observed [12] that another partial resolution can be achieved if the long-range part of the Ewald partition [13]

$r_{12}^{-1}=\frac{\operatorname{erfc}\left(\omega r_{12}\right)}{r_{12}}+\frac{\operatorname{erf}\left(\omega r_{12}\right)}{r_{12}}$

is expanded in series to yield

$\frac{\operatorname{erf}\left(\omega r_{12}\right)}{r_{12}}=\frac{2 \omega}{\sqrt{\pi}} \sum_{k=0}^{\infty} \frac{\left(-\omega^{2}\right)^{k}}{k !(2 k+1)}\left(r_{1}^{2}+r_{2}^{2}-2 \boldsymbol{r}_{1} \cdot \boldsymbol{r}_{2}\right)^{k}$

However, such an expansion is not fully satisfactory because it includes off-diagonal terms and, of course, because the short-range part remains unresolved. A similar partial resolution underpins the KWIK treatment [14-16] of the Coulomb operator.

In a recent communication [17], however, we have presented a more complete solution to the problem, showing that, given a complete set of functions $\left\{f_{k}(\boldsymbol{r})\right\}$ with the Coulomb-orthonormality property

$\left\langle f_{i}\left|r_{12}^{-1}\right| f_{j}\right\rangle=\delta_{i j}$

one obtains the Coulomb resolution (1) by choosing the $\phi_{k}(\boldsymbol{r})$ to be the Coulomb potentials of the $f_{k}(\boldsymbol{r})$.

We also showed that the functions

$f_{n l m}(\boldsymbol{r})=\frac{Y_{l m}(\boldsymbol{r})}{\pi \sqrt{2}} \int_{0}^{\infty} x^{2} h_{n}(x) j_{l}(x r) d x$

are complete and Coulomb-orthonormal, if the $j_{l}$ are spherical Bessel functions [11] and $\left\{h_{n}\right\}$ is a set of functions that are complete and orthonormal on $[0, \infty)$. Moreover, the Coulomb potential of $f_{n l m}(\boldsymbol{r})$ is

$\phi_{n l m}(\boldsymbol{r})=2 \sqrt{2} V_{n l}(r) Y_{l m}(\boldsymbol{r})$

where the radial potentials are defined by

$V_{n l}(r)=\int_{0}^{\infty} h_{n}(x) j_{l}(x r) d x$

and comparison with (4) then reveals that

$\frac{4 l+2}{\pi} \sum_{n=0}^{\infty} V_{n l}\left(r_{1}\right) V_{n l}\left(r_{2}\right)=\frac{r_{<}^{l}}{r_{>}^{l+1}}$

There is an infinite variety of generators $h_{n}(x)$ and each yields a valid Coulomb resolution (1). In principle, any one of these can be chosen but, in practice, the choice is guided by computational considerations. In [17], we chose the discrete Hermite generator

$h_{n}(x)=\frac{(2 / \pi)^{1 / 4}}{2^{n} \sqrt{(2 n) !}} H_{2 n}\left(\frac{x}{\sqrt{2}}\right) \exp \left(-\frac{x^{2}}{4}\right)$

but this leads to radial potentials that are numerically difficult. Continuous generators are also possible, for example the Dirac generator is

$h_{n}(x)=\delta(x-n)$

where the index $n$ runs over all positive real numbers. The potentials (10) from this generator are simply the spherical Bessel functions

$V_{n l}(r)=j_{l}(n r)$

and the resulting Coulomb resolution is therefore

$r_{12}^{-1}=8 \int_{0}^{\infty} \sum_{l m} j_{l}\left(n r_{1}\right) Y_{l m}\left(\boldsymbol{r}_{1}\right) j_{l}\left(n r_{2}\right) Y_{l m}\left(\boldsymbol{r}_{2}\right) d n$
This is elegant but it requires an integration, rather than a sum, over $n$ and is therefore less convenient from a computational point of view. In the present paper, we consider an alternative generator based on the Laguerre polynomials and show that this has several computational advantages. We use atomic units throughout.

\section{The Laguerre generator}

We define the Laguerre generator to be

$h_{n}(x)=\sqrt{2} L_{n}(2 x) \exp (-x)$

$(n=0,1,2, \ldots)$ which provides a complete orthonormal set on $[0, \infty)$.

By using the integral representation [11]

$j_{l}(z)=\frac{(-i)^{l}}{2} \int_{-1}^{1} \exp (i z t) P_{l}(t) d t$

(where $P_{l}$ is a Legendre polynomial), the radial potential (10) can be recast as

$V_{n l}(r)=\sqrt{2}(-i)^{l} \frac{z}{2} \int_{-1}^{1} \frac{(-z-t)^{n}}{(z-t)^{n+1}} P_{l}(t) d t$

where $z=(i r)^{-1}$. Each potential is a finite sum of elementary functions.

The $l=0$ potentials are given by

$V_{n 0}(r)=\sqrt{2} \frac{\theta}{r}\left[1+\sum_{k=1}^{n}(-1)^{k} \frac{\sin 2 k \theta}{k \theta}\right]$

where $\theta=\tan ^{-1} r$. Eq. (19) provides a simple and stable recursive scheme for computing the $V_{n 0}(r)$ and Fig. 1 illustrates low-order examples of these.

The $n=0$ potentials are given by

$V_{0 l}(r)=\sqrt{2} i^{l}(i / r) Q_{l}^{0}(i / r)$

where $Q_{l}^{k}$ is an associated Legendre function of the second kind [11]. These potentials are everywhere non-negative and Fig. 2 illustrates low-order examples of these.

The Laguerre generators (16) and spherical Bessel functions (17) possess the recursive properties [11]

$\int\left[h_{n+1}(x)+h_{n}(x)\right] d x=h_{n}(x)-h_{n+1}(x)$

$(2 l+1) \frac{d}{d z} j_{l}(z)=l j_{l-1}(z)-(l+1) j_{l+1}(z)$

and integration by parts of (10) therefore gives

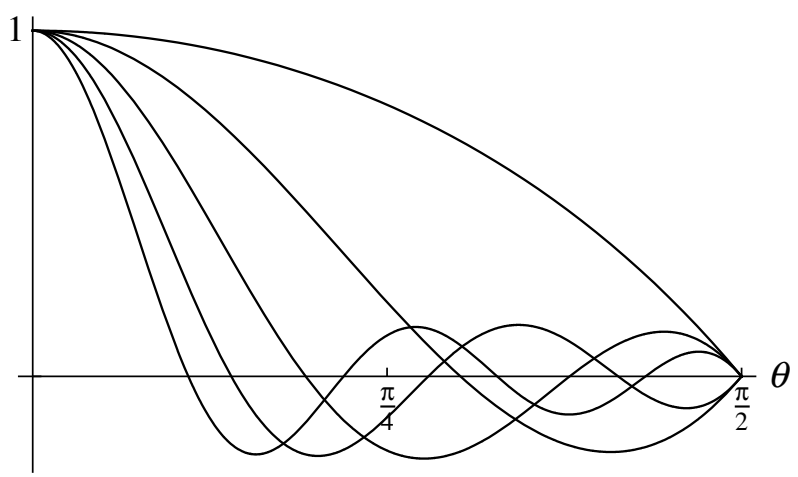

Fig. 1. $(-1)^{n} V_{n 0}(r)$ for $n=0,1,2,3,4 .(r \equiv \tan \theta)$. 


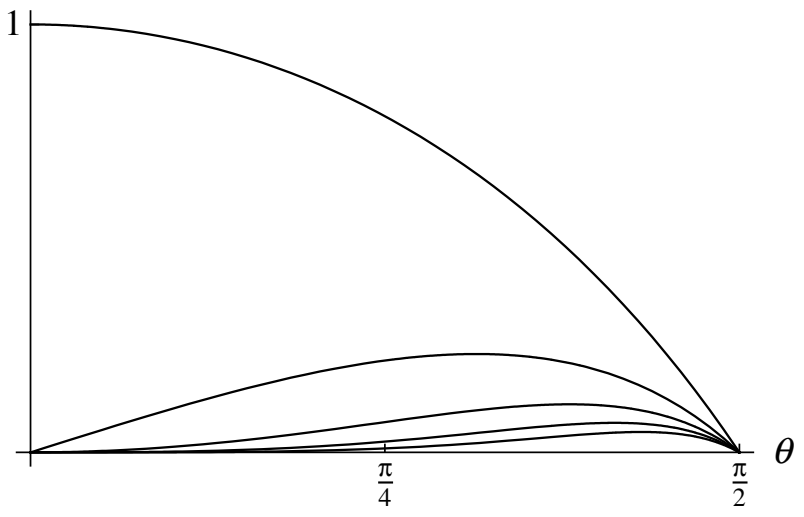

Fig. 2. $V_{0 l}(r)$ for $l=0,1,2,3,4$. $(r \equiv \tan \theta)$.

$$
\begin{aligned}
V_{n+1, l}+V_{n, l}= & \int_{0}^{\infty}\left[h_{n+1}(x)+h_{n}(x)\right] j_{l}(r x) d x \\
= & \frac{r}{2 l+1} \int_{0}^{\infty}\left[h_{n+1}(x)-h_{n}(x)\right] \times\left[l j_{l-1}(r x)\right. \\
& \left.-(l+1) j_{l+1}(r x)\right] d x \\
= & \frac{r}{2 l+1}\left[l\left(V_{n+1, l-1}-V_{n, l-1}\right)-(l+1)\left(V_{n+1, l+1}-V_{n, l+1}\right)\right]
\end{aligned}
$$

This can be used for the recursive construction of the radial potentials, beginning from the boundary values (19) and (20).

For small $r$, one can show

$V_{n l}(r) \sim \sqrt{\frac{\pi}{2}} \frac{\Gamma(l+1)}{\Gamma\left(l+\frac{3}{2}\right)} F(-n, l+1,1,2)(r / 2)^{l}$

where $F(a, b, c, x)$ is the hypergeometric function [11]. For large $r$, one can show

$V_{n l}(r) \sim \sqrt{\frac{\pi}{2}} \frac{\Gamma\left(\frac{l+1}{2}\right)}{\Gamma\left(\frac{l+2}{2}\right)} r^{-1}$

showing that every potential decays as $O\left(r^{-1}\right)$.

Eq. (11) can be used to assess the accuracy of truncated versions of the Coulomb resolution. For example, if we freeze $r_{1}=1$ and then truncate (11) after $n=10$, we obtain, for $l=0$,

$\frac{2}{\pi} \sum_{n=0}^{10} V_{n 0}\left(r_{1}\right) V_{n 0}\left(r_{2}\right) \approx \begin{cases}1 & 0<r_{2}<1 \\ 1 / r_{2} & 1<r_{2}<\infty\end{cases}$

which is illustrated in Fig. 3. We see that this 11-term approximation is well-behaved for all $r_{2}$ but that it is least accurate near $r_{1}=r_{2}$ (i.e. near $\theta=\pi / 4$ ).

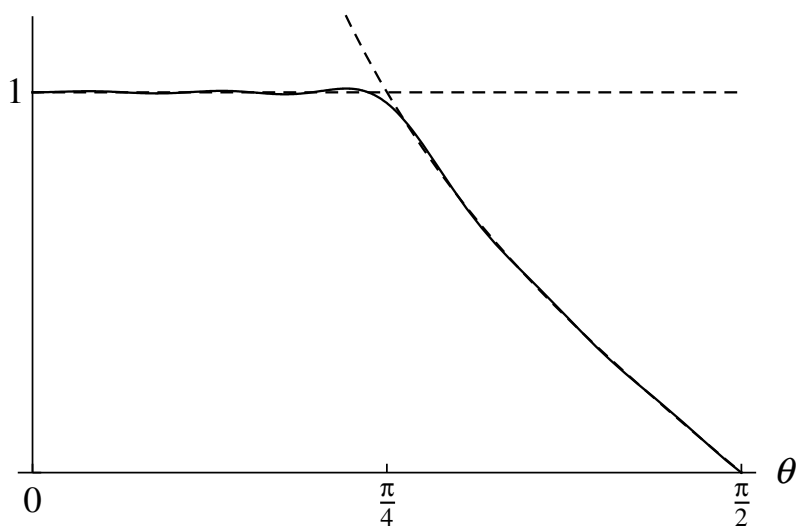

Fig. 3. Comparison of $\frac{2}{\pi} \sum_{n=0}^{10} V_{n 0}(1) V_{n 0}(r)$ (solid) with 1 and $1 / r$ (dashed).

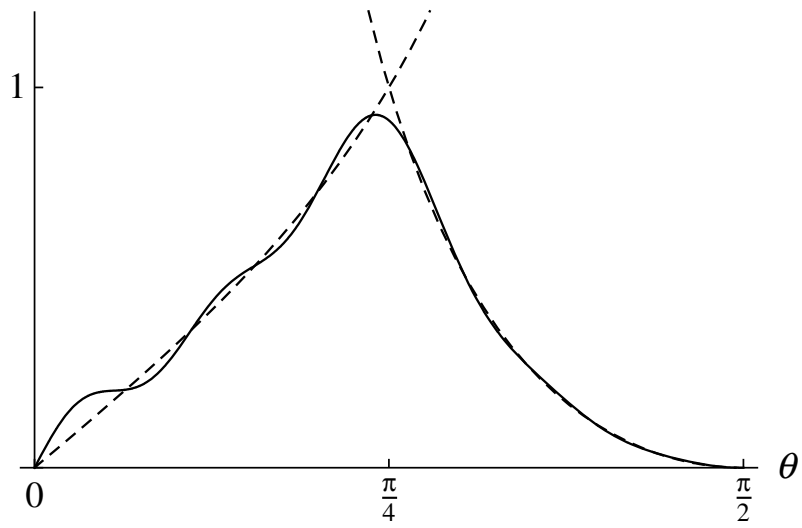

Fig. 4. Comparison of $\frac{6}{\pi} \sum_{n=0}^{10} V_{n 1}(1) V_{n 1}(r)$ (solid) with $r$ and $1 / r^{2}$ (dashed).

The analogous analysis for $l=1$ yields

$\frac{6}{\pi} \sum_{n=0}^{10} V_{n 1}\left(r_{1}\right) V_{n 1}\left(r_{2}\right) \approx \begin{cases}r_{2} & 0<r_{2}<1 \\ 1 / r_{2}^{2} & 1<r_{2}<\infty\end{cases}$

and this is illustrated in Fig. 4. This approximation is less accurate than that for $l=0$, particularly for $r_{2}<r_{1}$.

\section{Overlap integrals}

To apply the resolution (1) to problems in which a charge density is expanded in a basis, we need the overlap integral $\left\langle g \mid \phi_{n l m}\right\rangle$ between a basis function $g(|\boldsymbol{r}-\boldsymbol{R}|)$ and a potential $\phi_{n l m}(\boldsymbol{r})$.

Substituting (9) and invoking the Convolution theorem, we obtain

$\left\langle g \mid \phi_{n l m}\right\rangle=\left\langle h_{n} \mid \gamma_{l m}\right\rangle$

where we have introduced the "structure harmonics"

$\gamma_{l m}(x)=2 \sqrt{2} Y_{l m}(\boldsymbol{R}) j_{l}(R x) \hat{g}(x)$

and $\hat{g}(x)$ is the Fourier transform of $g(r)$. For example, in a Gaussian basis, we find

$g(\boldsymbol{r})=(\zeta / \pi)^{3 / 2} \exp \left(-\zeta r^{2}\right)$

$\gamma_{l m}(x)=2 \sqrt{2} Y_{l m}(\boldsymbol{R}) j_{l}(R x) \exp \left(-\frac{x^{2}}{4 \zeta}\right)$

and, in a Slater basis, we find

$g(\boldsymbol{r})=\left(\zeta^{3} / 8 \pi\right) \exp (-\zeta r)$

$\gamma_{l m}(x)=2 \sqrt{2} Y_{l m}(\boldsymbol{R}) j_{l}(R x)\left(1+(x / \zeta)^{2}\right)^{-2}$

Recalling that the $h_{n}$ form an orthonormal basis, we see from (28) that the auxiliary integral $\left\langle g \mid \phi_{n l m}\right\rangle$ is simply the projection of $\gamma_{l m}$ into the $h_{n}$ basis. We will use this below to understand the convergence behaviour of the resolution.

\section{Resolved Coulomb and exchange energies}

The Coulomb energy

$E_{\mathrm{J}}=\frac{1}{2}\left\langle\rho\left|r_{12}^{-1}\right| \rho\right\rangle$

of a charge density $\rho(\boldsymbol{r})$ is its classical self-interaction. Substituting the Coulomb resolution (1) yields the alternative expression

$E_{\mathrm{J}}=\frac{1}{2} \sum_{n l m}^{\infty}\left\langle\rho \mid \phi_{n l m}\right\rangle^{2}=\sum_{n l m}^{\infty} E_{\mathrm{J}}^{n l m}$ 
The exchange energy

$E_{\mathrm{K}}=\sum_{i j}^{\mathrm{occ}}\left\langle\psi_{i} \psi_{j}\left|r_{12}^{-1}\right| \psi_{i} \psi_{j}\right\rangle$

of a set of occupied orbitals $\psi_{i}$ is a consequence of the antisymmetry principle. Substituting the Coulomb resolution (1) yields the alternative expression

$E_{\mathrm{K}}=\sum_{i j}^{\mathrm{occ}} \sum_{n l m}^{\infty}\left\langle\psi_{i} \psi_{j} \mid \phi_{n l m}\right\rangle^{2}=\sum_{n l m}^{\infty} E_{\mathrm{K}}^{n l m}$

Because the $E_{\mathrm{J}}^{n l m}$ and $E_{\mathrm{K}}^{n l m}$ are always non-negative, the sums in (35) and (37) converge from below.

\section{Numerical results}

\subsection{Coulomb energy of a hydrogenic ion}

The ground-state electron density of a hydrogenic ion with nuclear charge $Z$ is

$\rho(\boldsymbol{r})=\left(Z^{3} / \pi\right) \exp (-2 Z r)$

and its Coulomb energy is $E_{\mathrm{J}}=5 Z / 16$. Its only non-vanishing structure harmonic (29) is

$\gamma_{00}(x)=\sqrt{\frac{2}{\pi}} \frac{1}{\left(1+x^{2} / 4 Z^{2}\right)^{2}}$

and, because this is a smooth function, its expansion coefficients $\left\langle h_{n} \mid \gamma_{00}\right\rangle$ in the $h_{n}(x)$ basis decay rapidly [18] with increasing $n$.

The results in Table 1 reflect this, revealing that the $E_{J}^{n 00}$ decrease more or less exponentially with $n$. However, whereas the $\mathrm{H}$ atom (i.e. $Z=1$ ) terms drop below the millihartree level at $n=3$ and below the microhartree level at $n=9$, those for $\mathrm{B}^{+4}$ ion (i.e. $Z=5$ ) do not reach the millihartree level until $n=10$.

\begin{tabular}{|c|c|c|c|c|c|}
\hline$n$ & $Z=1$ & $Z=2$ & $Z=3$ & $Z=4$ & $Z=5$ \\
\hline 0 & 0.5 & 0.3 & 0.3 & 0.2 & 0.2 \\
\hline 1 & 2.0 & 0.8 & 0.5 & 0.4 & 0.3 \\
\hline 2 & 2.8 & 1.9 & 1.1 & 0.7 & 0.6 \\
\hline 3 & 3.4 & 3.0 & 1.7 & 1.1 & 0.9 \\
\hline 4 & 5.4 & 3.4 & 2.3 & 1.6 & 1.2 \\
\hline 5 & 6.1 & 4.0 & 2.8 & 2.1 & 1.6 \\
\hline 6 & 5.1 & 4.6 & 3.3 & 2.5 & 1.9 \\
\hline 7 & 5.5 & 4.9 & 3.7 & 2.9 & 2.3 \\
\hline 8 & 5.8 & 5.4 & 4.1 & 3.2 & 2.6 \\
\hline 9 & 6.6 & 5.7 & 4.4 & 3.5 & 2.9 \\
\hline 10 & 7.2 & 6.1 & 4.7 & 3.8 & 3.2 \\
\hline
\end{tabular}

The reason for this $Z$ dependence is simple. As $Z$ increases, the density (38) of the hydrogenic ion contracts toward the nucleus, the structure harmonic (39) becomes more diffuse and, as a result, the Laguerre generator $h_{n}(x)$ in (16) becomes a less effective expansion basis for $\gamma_{00}(x)$. Of course, the behaviour for $\mathrm{B}^{+4}$ would mimic that of $\mathrm{H}$ if the Laguerre generator were simply dilated by a factor of five.

\subsection{Coulomb energy of a hydrogen molecule}

A model density for a hydrogen molecule is

$\rho(\boldsymbol{r})=\frac{\exp (-2|\boldsymbol{r}-\boldsymbol{R} / 2|)+\exp (-2|\boldsymbol{r}+\boldsymbol{R} / 2|)}{\pi}$

and it can be shown [19] that its Coulomb energy is

$E_{\mathrm{J}}=\frac{5}{8}+\frac{1}{R}-\left(\frac{1}{R}+\frac{11}{8}+\frac{3 R}{4}+\frac{R^{2}}{6}\right) \exp (-2 R)$

If the nuclei are placed at $(0,0, \pm R / 2)$, all of the structure harmonics vanish, except those with even $l$ and $m=0$. In those non-vanishing cases, we have

$\gamma_{l 0}(x)=\sqrt{\frac{8(2 l+1)}{\pi}} \frac{j_{l}(R x / 2)}{\left(1+x^{2} / 4\right)^{2}}$

The experimental bond length of $\mathrm{H}_{2}$ is $R_{\mathrm{eq}}=1.4$. At the compressed bond length $R=1$, we obtain

$E_{\mathrm{J}}=\frac{13}{8}-\frac{79}{24} \exp (-2)=1.179521 \ldots$

and, at the stretched bond length $R=2$, we obtain

$E_{\mathrm{J}}=\frac{9}{8}-\frac{97}{24} \exp (-4)=1.050974 \ldots$

Thus, the Coulomb energy drops by 129 millihartrees.

The resolutions of the energies at these two bond lengths are shown in Table 2. Examination and comparison of these data reveal a number of interesting aspects of the properties of the Coulomb resolution.

Because the density (40) has $D_{\infty h}$ symmetry, the $E_{\mathrm{J}}^{n l m}$ are nonzero if, and only if, $l$ is even and $m=0$. The contributions decrease rapidly with increasing $l$ but less so at longer $R$. For example, the fraction of $E_{\mathrm{J}}$ recovered by the $l=0$ terms alone is $99.7 \%$ and $96.9 \%$ for $R=1$ and $R=2$, respectively.

Fig. 5 shows the electron density (40) for $R=2$. It is clear that the density itself is far from spherically symmetric and one may ask why such a high proportion $(\approx 97 \%)$ of the Coulomb energy is recovered at the $l=0$ level. This felicitous outcome follows from the Coulomb orthonormality that underlies the resolution (1).

Table 2

$-\log \left(E_{\mathrm{J}}^{n l 0}\right)$ for the $\mathrm{H}_{2}$ molecule with bond lengths $R=1$ and $R=2$.

\begin{tabular}{|c|c|c|c|c|c|c|c|c|c|c|c|c|}
\hline \multirow[t]{2}{*}{$n$} & \multicolumn{6}{|l|}{$R=1$} & \multicolumn{6}{|l|}{$R=2$} \\
\hline & $l=0$ & $l=2$ & $l=4$ & $l=6$ & $l=8$ & $l=10$ & $l=0$ & $l=2$ & $l=4$ & $l=6$ & $l=8$ & $l=10$ \\
\hline 0 & -0.1 & 3.2 & 6.7 & 10.2 & 13.6 & 16.9 & 0.0 & 2.1 & 4.6 & 7.0 & 9.3 & 11.5 \\
\hline 1 & 1.8 & 2.6 & 5.5 & 8.6 & 11.7 & 14.7 & 5.6 & 1.7 & 3.5 & 5.5 & 7.5 & 9.5 \\
\hline 2 & 1.9 & 3.2 & 5.1 & 7.7 & 10.4 & 13.1 & 1.7 & 3.1 & 3.3 & 4.7 & 6.4 & 8.1 \\
\hline 3 & 2.8 & 6.9 & 5.2 & 7.1 & 9.5 & 11.9 & 2.3 & 2.9 & 4.3 & 4.6 & 5.8 & 7.2 \\
\hline 4 & 4.0 & 5.1 & 5.5 & 6.9 & 8.8 & 11.0 & 3.7 & 4.3 & 4.9 & 5.1 & 5.6 & 6.6 \\
\hline 5 & 5.0 & 7.1 & 5.9 & 6.8 & 8.3 & 10.3 & 4.9 & 5.7 & 5.3 & 10.9 & 5.9 & 6.4 \\
\hline 6 & 4.5 & 6.2 & 6.6 & 6.9 & 8.1 & 9.7 & 4.4 & 6.2 & 8.4 & 6.0 & 7.2 & 6.6 \\
\hline 7 & 4.8 & 7.6 & 8.0 & 7.1 & 7.9 & 9.3 & 4.5 & 5.7 & 6.3 & 6.7 & 6.9 & 7.4 \\
\hline 8 & 5.1 & 6.8 & 8.5 & 7.5 & 7.9 & 8.9 & 4.8 & 5.5 & 7.2 & 7.2 & 7.0 & 7.9 \\
\hline 9 & 5.8 & 7.4 & 8.0 & 8.3 & 8.0 & 8.8 & 5.4 & 6.0 & 6.9 & 7.1 & 9.9 & 7.5 \\
\hline 10 & 6.7 & 9.3 & 8.4 & 11.4 & 8.3 & 8.7 & 6.3 & 7.4 & 8.1 & 8.7 & 7.6 & 8.5 \\
\hline All & -0.1 & 2.4 & 4.6 & 6.2 & 7.2 & 8.0 & 0.0 & 1.5 & 3.0 & 4.3 & 5.2 & 6.0 \\
\hline
\end{tabular}




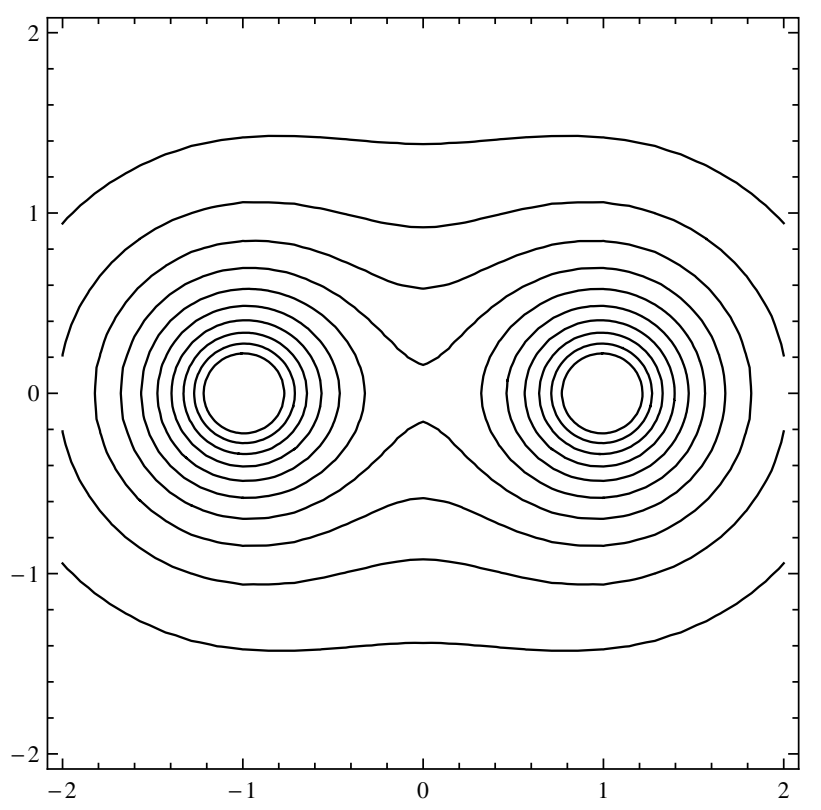

Fig. 5. $\rho(\boldsymbol{r})$ in $\mathrm{H}_{2}$ with bond length $R=2$.

Because it produces a diagonal resolution, there are no cross terms and $E_{\mathrm{J}}$ is resolved into a monopole-monopole $(l=0)$ component, a quadrupole-quadrupole $(l=2)$ component, a hexadecapole-hexadecapole $(l=4)$ component, and so forth. Unsurprisingly, these decrease rapidly and, for example, the inclusion of terms up to only $l=4$ (for $R=1$ ) or $l=8$ (for $R=2$ ) are already sufficient to estimate $E_{\mathrm{J}}$ with microhartree accuracy.

Such rapid convergence bodes well for the computational usefulness of the Coulomb resolution in more complicated applications.

\subsection{Exchange energy of a beryllium atom}

Using Slater's rules for the exponents, we obtain the groundstate beryllium orbitals

$\psi_{1 s}(\boldsymbol{r})=\sqrt{\frac{50653}{1000 \pi}} \exp \left(-\frac{37}{10} r\right)$

$\psi_{2 s}(\boldsymbol{r})=\sqrt{\frac{300}{1099 \pi}}\left(1-\frac{47}{30} r\right) \exp (-r)$

and the resulting exchange energy is

$E_{\mathrm{K}}=\frac{187504460349243315}{70912800972699392}$

The structure harmonics for the orbital products $\psi_{i} \psi_{j}$ in (37) are all smooth and the results in Table 3 reflect this, showing that the $E_{\mathrm{K}}^{n 00}$ fall below one milli- and microhartree at $n=8$ and 21 , respectively.
Table 3

$-\log \left(E_{\mathrm{K}}^{n 00}\right)=\varepsilon_{n}$ for the Be atom.

\begin{tabular}{lrllllll}
\hline$n$ & $\varepsilon_{n}$ & $n$ & $\varepsilon_{n}$ & $n$ & $\varepsilon_{n}$ & $n$ & $\varepsilon_{n}$ \\
\hline 0 & -0.1 & 11 & 4.0 & 22 & 6.2 & 33 & 7.6 \\
1 & 0.1 & 12 & 4.2 & 23 & 6.4 & 34 & 7.7 \\
2 & 0.5 & 13 & 4.5 & 24 & 6.5 & 35 & 7.8 \\
3 & 1.0 & 14 & 4.8 & 25 & 6.6 & 36 & 7.9 \\
4 & 1.5 & 15 & 5.0 & 26 & 6.8 & 37 & 8.0 \\
5 & 1.9 & 16 & 5.2 & 27 & 6.9 & 38 & 8.0 \\
6 & 2.4 & 17 & 5.4 & 28 & 7.0 & 39 & 8.1 \\
7 & 2.8 & 18 & 5.5 & 29 & 7.1 & 40 & 8.2 \\
8 & 3.1 & 19 & 5.7 & 30 & 7.2 & 41 & 8.3 \\
9 & 3.4 & 20 & 5.9 & 31 & 7.4 & 42 & 8.4 \\
10 & 3.7 & 21 & 6.0 & 32 & 7.5 & 43 & 8.5 \\
\hline
\end{tabular}

The convergence behaviour is similar to that for the $\mathrm{Be}^{+3}$ ion (i.e. $Z=4$ ) in Table 1 and this seems very reasonable when it is realized that it is the $\psi_{1 s} \psi_{1 s}$ product, which is almost the same in Be and $\mathrm{Be}^{+3}$, that determines the convergence rate.

\section{Concluding remarks}

We have explored a new resolution of the Coulomb operator that seems more suitable for numerical work than the resolution introduced in our earlier communication [17]. We have applied it to the calculation of Coulomb and exchange energies in a few very simple systems and we have observed encouraging convergence behaviour that can be traced to the fact that the resolution provides a diagonal representation of the operator. Further work, focusing on analytical expressions for the overlap integrals (28) and applications to larger systems will be reported elsewhere.

\section{References}

[1] N.H.F. Beebe, J. Linderberg, Int. J. Quant. Chem. 12 (1977) 683.

[2] H. Koch, A. Sanchez de Meras, T.B. Pedersen, J. Chem. Phys. 118 (2003) 9481.

[3] F. Aquilante, R. Lindh, T.B. Pedersen, J. Chem. Phys. 127 (2007) 114107.

[4] S.R. Chinnamsetty, M. Espig, B.N. Khoroskij, W. Hackbusch, H.-J. Flad, J. Chem. Phys. 127 (2007) 84110.

[5] B.I. Dunlap, J. Connolly, J. Sabin, J. Chem. Phys. 71 (1979) 3396.

[6] O. Vahtras, J. Almlöf, M. Feyereisen, Chem. Phys. Lett. 213 (1993) 514.

[7] K. Eichkorn, O. Treutler, H. Öhm, M. Häser, R. Ahlrichs, Chem. Phys. Lett. 240 (1995) 283.

[8] Y. Jung, A. Sodt, P.M.W. Gill, M. Head-Gordon, Proc. Natl. Acad. Sci. USA 102 (2005) 6692.

[9] F.R. Manby, P.J. Knowles, Phys. Rev. Lett. 87 (2001) 163001

[10] F.R. Manby, P.J. Knowles, A.W. Lloyd, J. Chem. Phys. 115 (2001) 9144.

[11] M. Abramowitz, I.E. Stegun, Handbook of Mathematical Functions, Dover, New York, 1972.

[12] P.M.W. Gill, Chem. Phys. Lett. 270 (1997) 193.

[13] P.P. Ewald, Ann. Phys. (Leipzig) 64 (1921) 253.

[14] J.P. Dombroski, S.W. Taylor, P.M.W. Gill, J. Phys. Chem. 100 (1996) 6272.

[15] R.D. Adamson, J.P. Dombroski, P.M.W. Gill, Chem. Phys. Lett. 254 (1996) 329.

[16] P.M.W. Gill, R.D. Adamson, Chem. Phys. Lett. 261 (1996) 105.

[17] S.A. Varganov, A.T.B. Gilbert, E. Deplazes, P.M.W. Gill, J. Chem. Phys. 128 (2008) 201104.

[18] J.P. Boyd, Chebyshev and Fourier Spectral Methods, second ed., Dover, New York, 2000.

[19] P.R. Wallace, Mathematical Analysis of Physical Problems, Dover, New York, 1984. 